Research/Technical Note

\title{
Analysis of Squeeze Films Between Rough Circular Plates Lubricated with Rabinowitsch Fluids
}

\author{
Udaya Pratap Singh \\ Department of Mathematics, Rajkiya Engineering College, Sonbhadra, India \\ Email address: \\ journals4phd@gmail.com
}

\section{To cite this article:}

Udaya Pratap Singh. Analysis of Squeeze Films Between Rough Circular Plates Lubricated with Rabinowitsch Fluids. American Journal of Mechanics and Applications. Vol. 7, No. 1, 2019, pp. 1-9. doi: 10.11648/j.ajma.20190701.11

Received: May 12, 2019; Accepted: June 10, 2019; Published: June 25, 2019

\begin{abstract}
The present theoretical work investigates the combined impacts of non-Newtonian (pseudoplastic and dilatant) lubricants and surface roughness on the performance of squeeze films lubrication between two rough circular plates. The modified Reynolds equation has been derived on the basis of Christensen's stochastic theory of hydrodynamic lubrication for rough surfaces. The lubricant model adopted for the analysis is Rabinowitsch fluid model - an experimentally verified fluid model for lubricated bearing systems. Two types of one-dimensional roughness patterns (radial and azimuthal) have been considered in the analysis. An asymptotic solution for squeeze film pressure, load carrying capacity and squeeze film time are obtained. The numerical results for dimensionless film pressure, load carrying capacity and film squeezing time have been calculated for various values of fluid and operating parameters. The results for dimensionless film pressure, load capacity and squeezing time of the lubricant film have been discussed with clear graphical presentation for different values of parameters of pseudoplasticity and roughness. It was observed that the radial roughness decreases the film pressure, load capacity and squeezing time of lubricant, while increased values of these properties were observed for azimuthal roughness. It was also observed that the pseudoplastic lubricants decrease the film pressure and load capacity, while the dilatant lubricants increase these properties. Also, the variations in these results are highly significant.
\end{abstract}

Keywords: Surface Roughness, Circular Plates, Squeeze Film, Non-newtonian Fluids

\section{Introduction}

Study of squeeze film behaviour between two approaching surfaces is essential in many engineering applications and other allied areas. The effects of non-Newtonian lubricants on various performance properties of squeeze film, annular disks and externally pressurized bearings have been studied by many researchers $[1,2]$. Bakker and Ostayen presented recess shape optimization of annular disks [3]. Effects of surface roughness on pressure distribution of spherical bearing lubricated with Ellis fluids was studied by Jurczak and Falicki [4]. Effects of non-Newtonian lubricants on the performance of porus thrust bearings was investigated by Walicka et al. [5]. Many investigators also used power-law and couple stress fluid models to analyse steady and dynamic properties of film lubricated bearing systems [6, 7]. Singh at el. adopted Rabinowitsch fluid model for theoretical investigation of performance properties of many film lubricated bearings in different lubrication regimes [8-11]. To study the characteristics of bearings lubricated with non-Newtonian lubricants, Rabinowitsch fluid model is one of the best and experimentally verified fluid models [12]. The properties of slider bearings lubricated with non-Newtonian Rabinowitsch type lubricants were studied by Hsu and Saibel and it was shown that the pressure distribution of pseudoplastic fluids decreases below that of Newtonian fluids [13].

In Rabinowitsch fluid model, the relationship between shearing stress and shearing strain rate is given as:

$$
\tau_{x y}+k \tau_{x y}^{3}=\mu \frac{\partial u}{\partial \bar{y}}
$$

where, $\mu$ is the initial viscosity of Newtonian fluid and $k$ is the non-linear factor responsible for non-Newtonian nature of the lubricant [12]. By using this cubic equation model, one can 
analyse the dilatant fluids for $k<0$, Newtonian fluids for $k=0$ and pseudoplastic fluids for $k>0$, respectively. In an experimental investigation, Wada and Hayashi obtained a range of values of $\mu$ and $k$. Later on, this fluid model was used by several investigators to study the properties of hydrodynamic and hydrostatic bearings lubricated with non-Newtonian properties [12]. Problems concerning hydrostatic journal bearings were investigated by Sharma et al. [14], Sinhasan and Sah [15] and, Bourgin and Gay [16]. Slider bearings were analysed by Hashimoto [17] and Lin [18]. In recent years, Singh et al. [10, 19-21] used Rabinowitsch fluid model to investigate the static and dynamic properties of various hydrodynamic, hydrostatic and squeeze film bearings. Singh et al. [22-24] expanded the span of this fluid model to study the peristaltic motion of physiological fluids and got excellent results. Recently, Walicka et al. [25] analysed curvilinear squeeze film bearings using stochastic model for hydrodynamic lubrication [26]. But none of the researchers used Rabinowitsch fluid model to study the performance of squeeze films between rough circular plates. The objective of the present paper is to extend the results of Rabinowitsch model to squeeze films between rough circular plates.

\section{Mathematical Formulation and Solution}

Consider a squeeze film configuration (Figure 1) between rough circular plates lubricated with a non-Newtonian fluid. The plates are approaching each other with a normal velocity $v=-\partial H / \partial t$. In present study, it is assumed that the thin film theory of hydrodynamic lubrication is applicable.

Under these assumptions, the equations governing the one dimensional flow of incompressible non-Newtonian fluids are given by:

$$
\frac{1}{r} \frac{\partial(r u)}{\partial r}+\frac{\partial v}{\partial y}=0
$$

$$
\begin{gathered}
\frac{\partial p}{\partial r}=\frac{\partial \tau_{x y}}{\partial y} \\
\frac{\partial p}{\partial y}=0
\end{gathered}
$$

The boundary conditions for velocity components are:

$$
\begin{gathered}
u=0, \quad v=0 \text { at } y=0 \\
u=0, \quad v=-\frac{\partial h}{\partial t} \text { at } y=h
\end{gathered}
$$

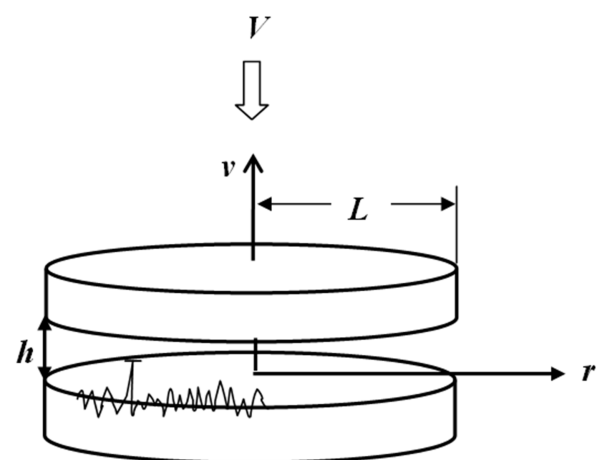

Figure 1. Schematics diagram of squeeze film between two rough circular plates.

Integration of the momentum equation (3) with respect to $y$ yields:

$$
\tau_{x y}=\frac{\partial p}{\partial r} y+I_{1}
$$

where $I_{1}$ is the constant of integration. Substituting the shear stress $\tau_{x y}$ into the equation (1) and integrating with respect to $y$, we get the velocity component as follows:

$$
u=\frac{1}{\mu}\left\{f \frac{y^{2}}{2}+I_{1} y+k\left(f^{3} \frac{y^{4}}{4}+f^{2} y^{3} I_{1}+\frac{3}{2} f y^{2} I_{1}^{2}+y I_{1}^{3}\right)\right\}+I_{2}
$$

where $f=\frac{\partial p}{\partial r}$ is the pressure gradient, $I_{1}=-\frac{1}{2} f \cdot h$ and $I_{2}=0$.

From equations (5-7), radial velocity component $u$ is obtained as:

$$
u=\frac{1}{\mu}\left\{\frac{1}{2} f F_{1}+k f^{3} F_{2}\right\}
$$

where,

$$
F_{1}=y(y-h), F_{2}=\frac{y^{4}}{4}-\frac{y^{3} h}{2}+\frac{3}{8} y^{2} h^{2}-\frac{y h^{3}}{8}
$$

Using Eq. (9) in Eq. (2) and integrating with respect to $y$ between the limits 0 to $h$, the modified Reynolds equation can be obtained as: 


$$
\frac{1}{r} \frac{\partial}{\partial r}\left[r\left\{h^{3}\left(\frac{\partial p}{\partial r}\right)+\frac{3 k}{20} h^{5}\left(\frac{\partial p}{\partial r}\right)^{3}\right\}\right]=12 \mu \frac{\partial h}{\partial t}
$$

Taking the stochastic average of Eq. (10) with respect to $f\left(h_{s}\right)$, the averaged modified Reynolds type equation can be obtained as:

$$
\frac{1}{r} \frac{\partial}{\partial r}\left[r\left\{E\left(h^{3}\right)\left(\frac{\partial E(p)}{\partial r}\right)+\frac{3 k}{20} E\left(h^{5}\right)\left(\frac{\partial E(p)}{\partial r}\right)^{3}\right\}\right]=12 \mu \frac{\partial h}{\partial t}
$$

where the expectancy operator $E(*)$ is defined by:

$$
E(*)=\int_{-\infty}^{\infty}(*) f\left(h_{s}\right) d h_{s}
$$

and the probability density function $f\left(h_{s}\right)$ of the stochastic film thickness $h_{s}$ is given as:

$$
f\left(h_{s}\right)= \begin{cases}\frac{35}{32 c^{7}}\left(c^{2}-h_{s}^{2}\right)^{3}, & -c<h_{s}<c \\ 0, & \text { elsewhere }\end{cases}
$$

where, $c$ is the parameter of roughness [26]. Following Christensen's theory, radial and azimuthal roughness patterns are adopted in the present theoretical analysis.

\subsection{One-Dimensional Radial Roughness}

For one-dimensional radial roughness pattern, the roughness is in the form of long narrow ridges and valleys running through $x$-direction. In this case, the non-dimensional stochastic film thickness is given as $H_{i}=h_{i}+h_{s}(\theta, \xi)$ for $i=1,2$; and the stochastic modified Reynolds equation (11) takes the form:

$$
\frac{1}{r} \frac{\partial}{\partial r}\left[r\left\{E\left(H^{3}\right)\left(\frac{\partial E(p)}{\partial r}\right)+\frac{3 k}{20} E\left(H^{5}\right)\left(\frac{\partial E(p)}{\partial r}\right)^{3}\right\}\right]=12 \mu \frac{d H}{d t}
$$

\subsection{One-Dimensional Azimuthal Roughness}

For one-dimensional azimuthal roughness pattern, the roughness is in the form of long narrow ridges and valleys running through $\theta$-direction. In this case, the non-dimensional stochastic film thickness is $H_{i}=h_{i}+h_{s}(r, \xi)$ for $i=1,2$; and the stochastic modified Reynolds equation (11) takes the form:

$$
\frac{1}{r} \frac{\partial}{\partial r}\left[r\left\{\frac{1}{E\left(\frac{1}{H^{3}}\right)}\left(\frac{\partial E(p)}{\partial r}\right)+\frac{3 k}{20} \frac{1}{E\left(\frac{1}{H^{5}}\right)}\left(\frac{\partial E(p)}{\partial r}\right)^{3}\right\}\right]=12 \mu \frac{d H}{d t}
$$

For axisymmetric flow, Eqs. (14-15) can be represented as:

$$
\frac{1}{r} \frac{\partial}{\partial r}\left[r\left\{G_{1}(H, c)\left(\frac{\partial E(p)}{\partial r}\right)+\frac{3 k}{20} G_{2}(H, c)\left(\frac{\partial E(p)}{\partial r}\right)^{3}\right\}\right]=12 \mu \frac{d H}{d t}
$$

where, 


$$
\begin{aligned}
& G_{1}(H, c)= \begin{cases}E\left(H^{3}\right) & ; \text { for radial roughness } \\
\left\{E\left(\frac{1}{H^{3}}\right)\right\}^{-1} & ; \text { for azimuthal roughness }\end{cases} \\
& E\left(H^{3}\right)=\frac{35}{32 c^{7}} \int_{-c}^{c} H^{3}\left(c^{2}-h_{s}^{2}\right)^{3} d h_{s} \\
& E\left(\frac{1}{H^{3}}\right)=\frac{35}{32 c^{7}} \int_{-c}^{c} \frac{\left(c^{2}-h_{s}^{2}\right)^{3}}{H^{3}} d h_{s}
\end{aligned}
$$

and,

$$
\begin{gathered}
G_{2}(H, c)=\left\{\begin{array}{l}
E\left(H^{5}\right) \quad ; \text { for radial roughness } \\
\left\{E\left(\frac{1}{H^{5}}\right)\right\}^{-1} ; \text { for azimuthal roughness }
\end{array}\right. \\
E\left(H^{5}\right)=\frac{35}{32 c^{7}} \int_{-c}^{c} H^{5}\left(c^{2}-h_{s}^{2}\right)^{5} d h_{s} \\
E\left(\frac{1}{H^{5}}\right)=\frac{35}{32 c^{7}} \int_{-c}^{c} \frac{\left(c^{2}-h_{s}^{2}\right)^{5}}{H^{5}} d h_{s}
\end{gathered}
$$

Introducing the dimensional quantities:

$$
r^{*}=\frac{r}{L}, p^{*}=\frac{E(p) h_{0}^{2}}{\mu L\left(-\frac{d H^{*}}{d t}\right)}, \beta=\frac{\mu^{2} k}{h_{0}^{2}\left(-\frac{d H^{*}}{d t}\right)^{2}}, H^{*}=\frac{H}{h_{0}}, C=\frac{c}{h_{0}}
$$

equation (16) takes the dimensionless form:

$\frac{\partial}{\partial r^{*}}\left[r^{*}\left\{G_{1}^{*}\left(H^{*}, C\right)\left(\frac{\partial p^{*}}{\partial r^{*}}\right)+\frac{3}{20} \beta G_{2}^{*}\left(H^{*}, C\right)\left(\frac{\partial p^{*}}{\partial r^{*}}\right)^{3}\right\}\right]=-12 r^{*}$

The squeeze film pressure can be perturbed as:

$$
p^{*}=p_{0}^{*}+\beta p_{1}^{*}
$$

Using Eq. (24) in the Reynolds equation (23) and neglecting higher order terms of $\beta$, following equations for squeeze film pressure $p_{0}^{*}$ and $p_{1}^{*}$ can be obtained:

$$
\begin{array}{r}
\frac{\partial}{\partial r^{*}}\left[r^{*}\left\{G_{1}^{*}\left(H^{*}, C\right) \frac{\partial p_{0}^{*}}{\partial r^{*}}\right\}\right]=-12 r^{*} \\
\frac{\partial}{\partial r^{*}}\left[r^{*}\left\{G_{1}^{*}\left(H^{*}, C\right) \frac{\partial p_{1}^{*}}{\partial r^{*}}+\frac{3}{20} G_{2}^{*}\left(H^{*}, C\right)\left(\frac{\partial p_{0}^{*}}{\partial r^{*}}\right)^{3}\right\}\right]=0
\end{array}
$$

Integrating Eqs. (24) and (25) with respect to $r^{*}$ with the boundary conditions:

$$
\begin{aligned}
\frac{d p^{*}}{d r^{*}} & =0 \text { at } r^{*}=0 \\
p^{*} & =0 \text { at } r^{*}=1
\end{aligned}
$$

the modified Reynolds type equation for squeeze film pressure is obtained as

$$
\begin{gathered}
\frac{d p_{0}^{*}}{d r^{*}}=\frac{-6 r^{*}}{G_{1}\left(H^{*}, C\right)} \\
\frac{d p_{1}^{*}}{d r^{*}}=\frac{162 G_{2}^{*}\left(H^{*}, C\right) r^{* 3}}{5 G_{1}^{* 4}\left(H^{*}, C\right)}
\end{gathered}
$$

From equations (24) and (27-30), expression for film pressure is obtained as:

$$
p^{*}=-\frac{3\left(r^{2}-1\right)}{G_{1}^{*}\left(H^{*}, C\right)}+\frac{81 \beta}{10} \frac{G_{2}^{*}\left(H^{*}, C\right)\left(r^{* 4}-1\right)}{G_{1}^{* 4}\left(H^{*}, C\right)}
$$


The average load carrying capacity is calculated as:

$$
E(W)=2 \pi \int_{0}^{L} r E(p) d r
$$

which takes the dimensionless form:

$$
W^{*}=2 \pi \int_{0}^{1} r^{*} p^{*} d r^{*}
$$

From equations (31) and (33), analytic expression for $W^{*}$ is obtained as:

$$
W^{*}=\frac{3 \pi}{2 G_{1}^{*}\left(H^{*}, C\right)}-\frac{27 \pi \beta}{5} \frac{G_{2}^{*}\left(H^{*}, C\right)}{G_{1}^{* 4}\left(H^{*}, C\right)}
$$

where,

$$
W^{*}=\frac{E(p) h_{0}^{2}}{\mu L\left(-\frac{d H^{*}}{d t}\right)}
$$

$$
t^{*}=\int_{h_{m}=h_{f}}^{1}\left(\frac{3 \pi}{2 G_{1}^{*}\left(H^{*}, C\right)}-\frac{27 \pi \beta}{5} \frac{G_{2}^{*}\left(H^{*}, C\right)}{G_{1}^{* 4}\left(H^{*}, C\right)}\right) d H^{*}
$$

where,

$$
t^{*}=\frac{E(W) h_{0}^{2}}{\mu L^{4}} t
$$

\section{Results and Discussion}

Based on the present theoretical analysis, the effects of non-Newtonian fluid and surface roughness on different properties of squeeze film between rough circular plates are presented. Results are analysed for different values of roughness parameter $C$ and pseudoplasticity parameter $\beta$.

Figure 2 shows the variation of non-dimensional pressure $p^{*}$ with non-dimensional radial coordinate $r^{*}$ for $h^{*}=0.4, C=$ 0.3 and different values of $\beta$. It is observed that the pressure $p^{*}$ decreases with increasing value of $r^{*}$ for each value of $\beta$ in both the cases of radial and azimuthal patterns. However, pressure for azimuthal roughness is always higher in comparison to that for radial roughness.

The squeezing time can be calculated as follows:

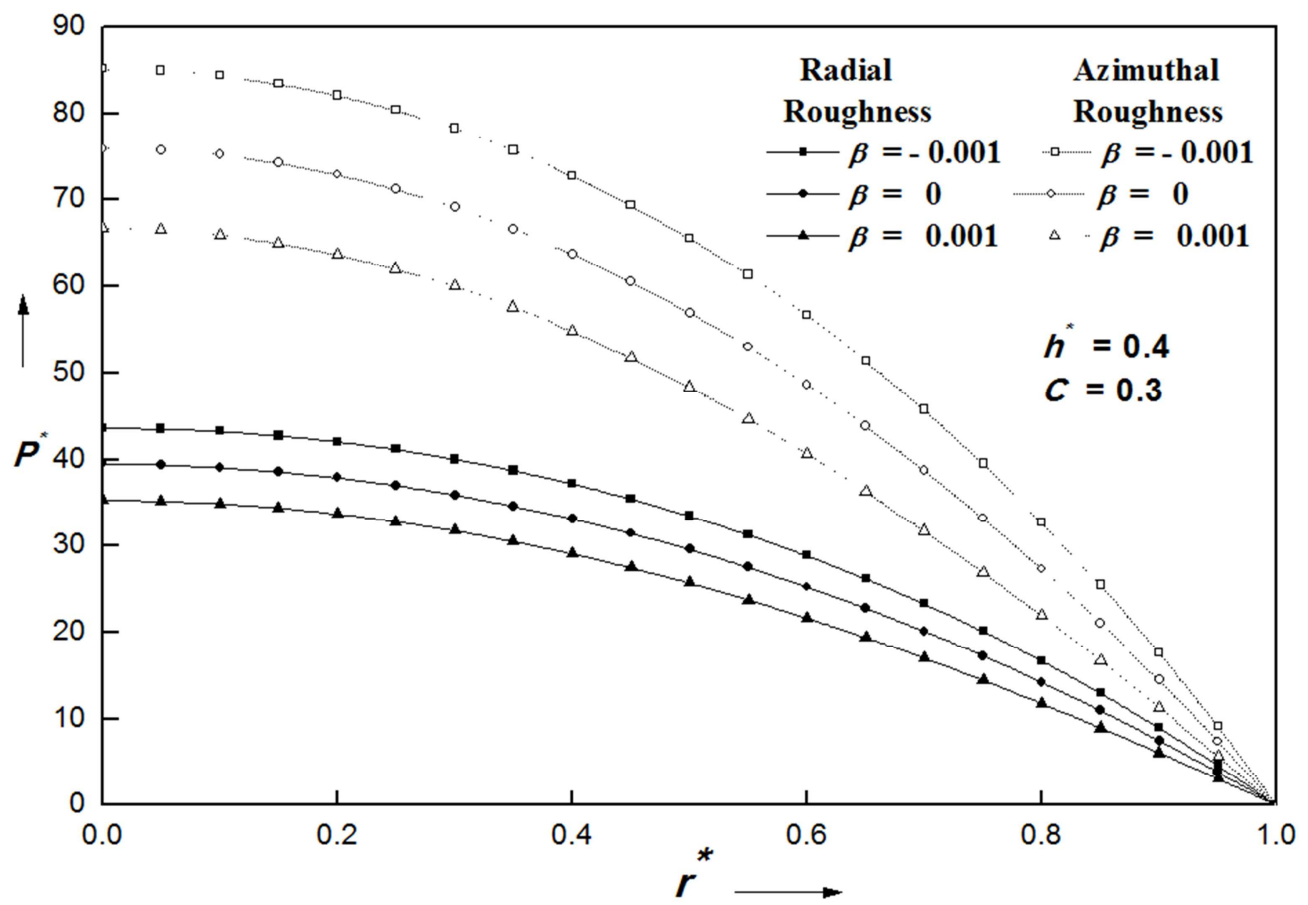

Figure 2. Variation of non-dimensional pressure $p^{*}$ with $r^{*}$ for different values of $\beta$ with $h^{*}=0.4$ and $C=0.3$.

The variation of dimensionless pressure $p^{*}$ with respect to $r^{*}$ for different values of $C$ with $\beta=0.001$ and $h^{*}=0.6$ is shown in Figure 3. It is seen that an increase in $C$ decreases the pressure for radial roughness, while increases the pressure for azimuthal roughness. The nature of variation of pressure sustains for all types of fluids. 


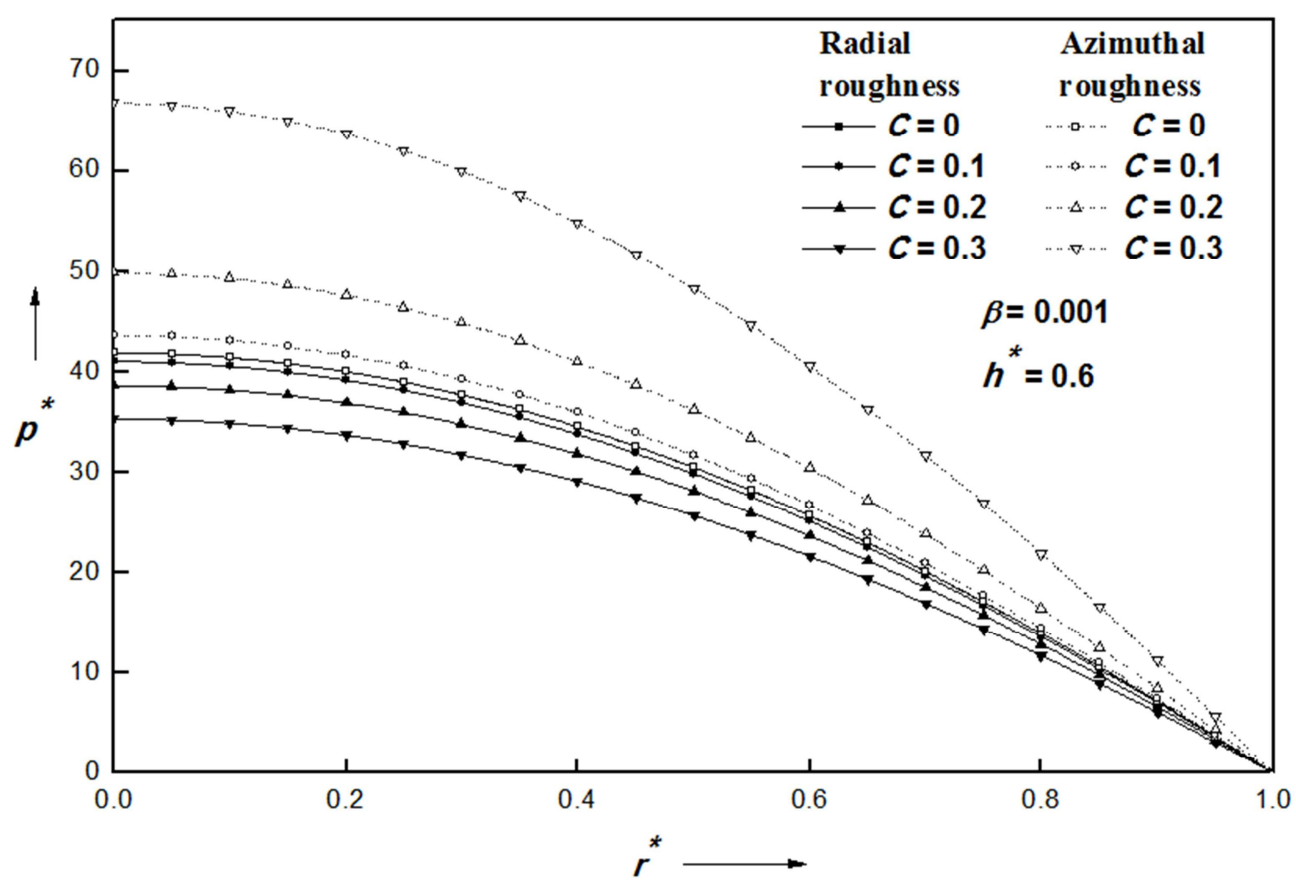

Figure 3. Variation of non-dimensional pressure $p^{*}$ with $r^{*}$ for different values of $C$ with $\beta=0.001$ and $h^{*}=0.6$.

Figure 4 depicts the variation of dimensionless load carrying capacity $W^{*}$ with dimensionless film thickness $h^{*}$ for different values of non-linear factor $\beta$ and specific value of roughness parameter $(C=0.3)$. It is observed that the load carrying capacity $W^{*}$ decreases with an increase of $h^{*}$, which satisfy the physical nature of the problem. In comparison to Newtonian fluids, the load capacity is higher for dilatant fluids, while lower for pseudoplastic fluids. The load capacity for azimuthal roughness is always higher than that for radial roughness.

Figure 5 shows the variation of non-dimensional load carrying capacity $W^{*}$ with $h^{*}$ for different values of $C$ and $\beta=$ 0.001 . It is observed that with an increase in value of $C$, the dimensionless load carrying capacity decreases in the case of radial roughness, while the load capacity increases in the case of azimuthal roughness.

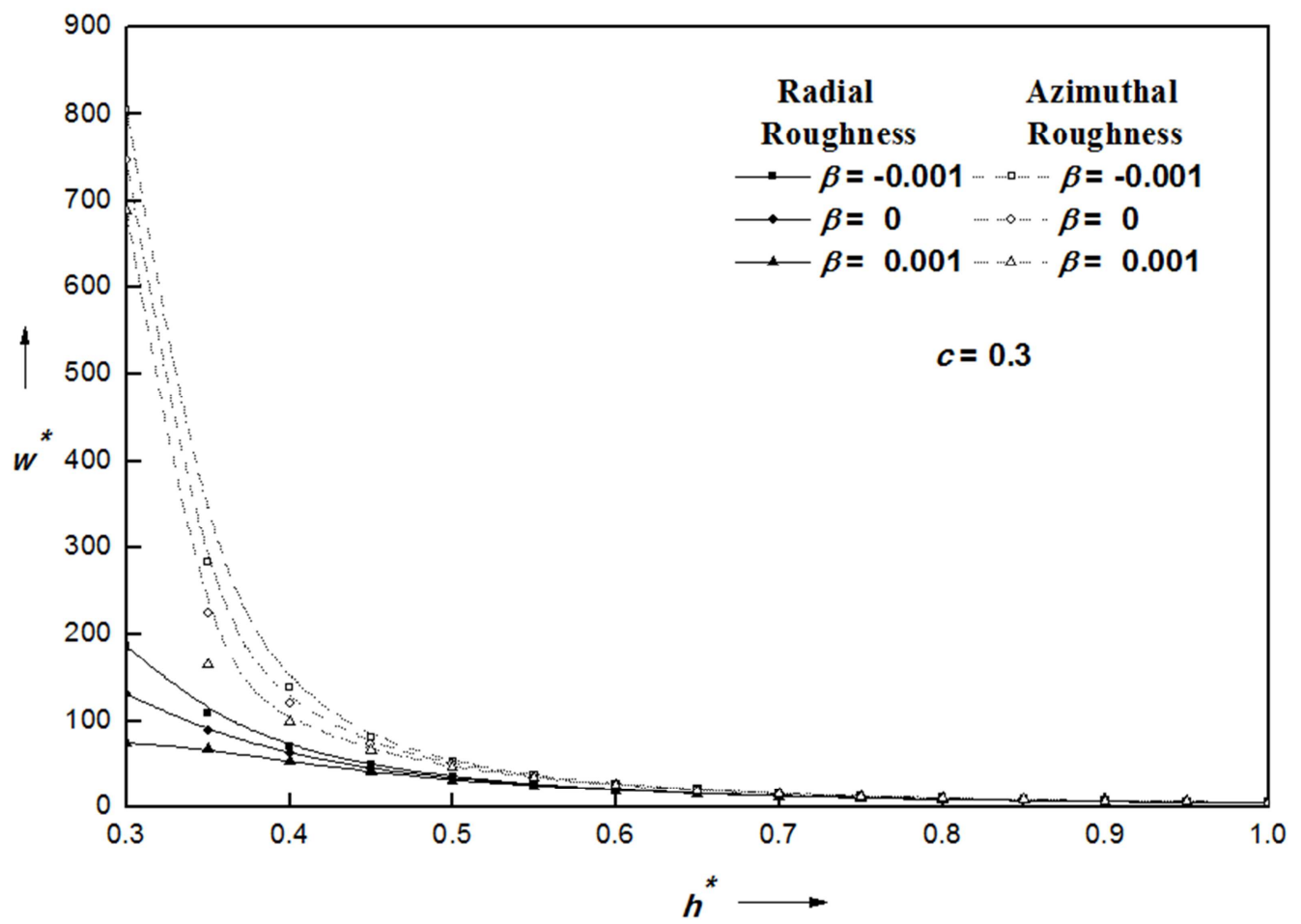

Figure 4. Variation of non-dimensional load carrying capacity $W^{*}$ with film thickness $h^{*}$ for different values of $\beta$ with $C=0.3$. 


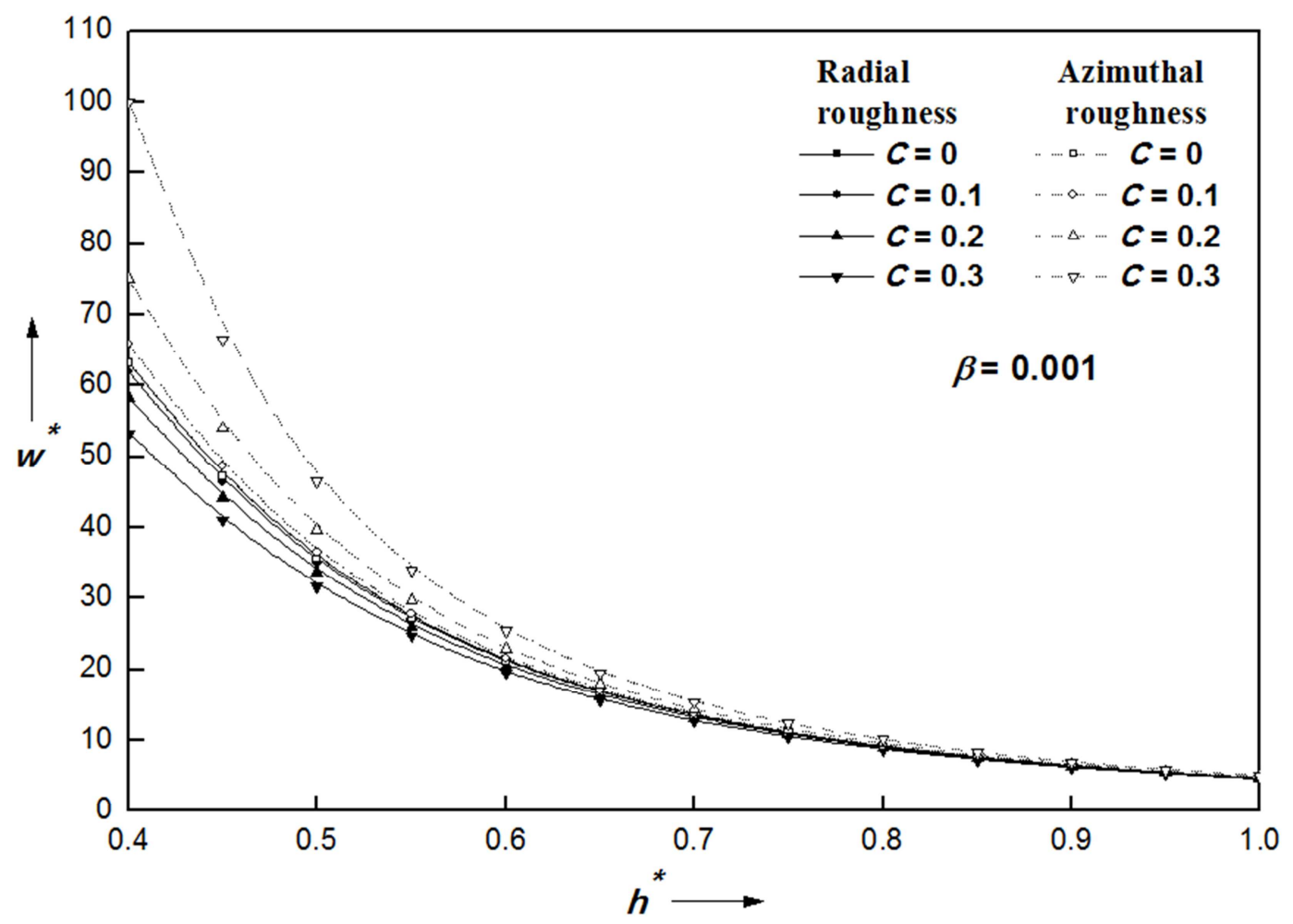

Figure 5. Variation of non-dimensional load carrying capacity $W^{*}$ with $h^{*}$ for different values of $C$ with $\beta=0.001$.

Figure 6 presents the variation of squeeze film time $t^{*}$ with dimensionless film thickness $h^{*}$ for different values of $\beta$ and $C$ $=0.3$. It is observed that the squeeze film time decreases with an increase in value of $h^{*}$. In comparison to Newtonian lubricants, squeezing time is smaller for pseudoplastic lubricants, while longer for dilatant lubricants. For all the types of lubricants, the squeezing time is longer for azimuthal roughness than that for radial roughness.

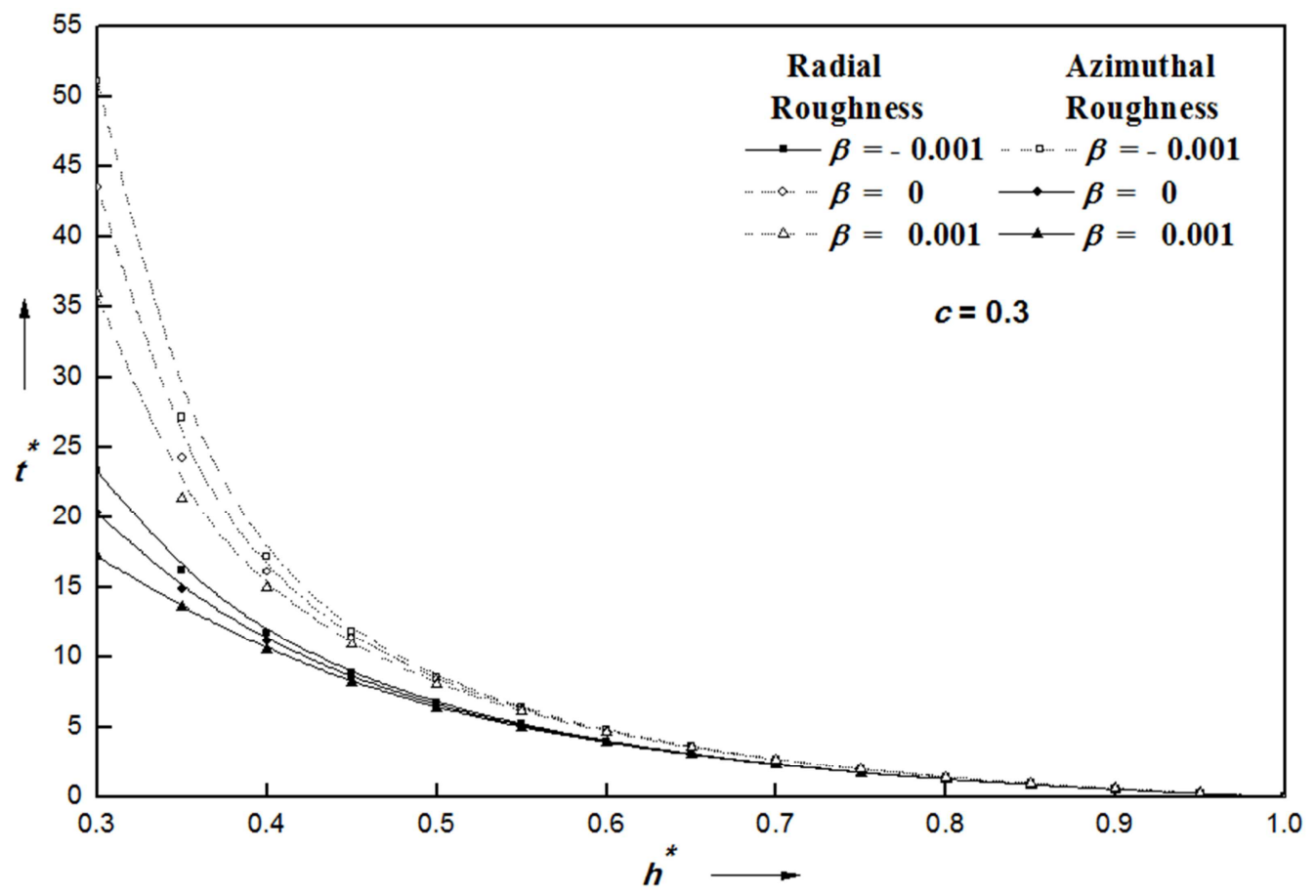

Figure 6. Variation of squeeze film time $t^{*}$ with film thickness $h^{*}$ for different values of $\beta$ with $C=0.3$.

The variation of squeeze film time $t^{*}$ with $h^{*}$ for different values of $C$ is shown in Figure 7. It is easy to observe that with the increase in the value of $C$, the squeezing time decreases for radial roughness and decreases for azimuthal roughness. The variation of squeezing time with amount and nature of surface roughness is in agreement with physical nature of the problem. 


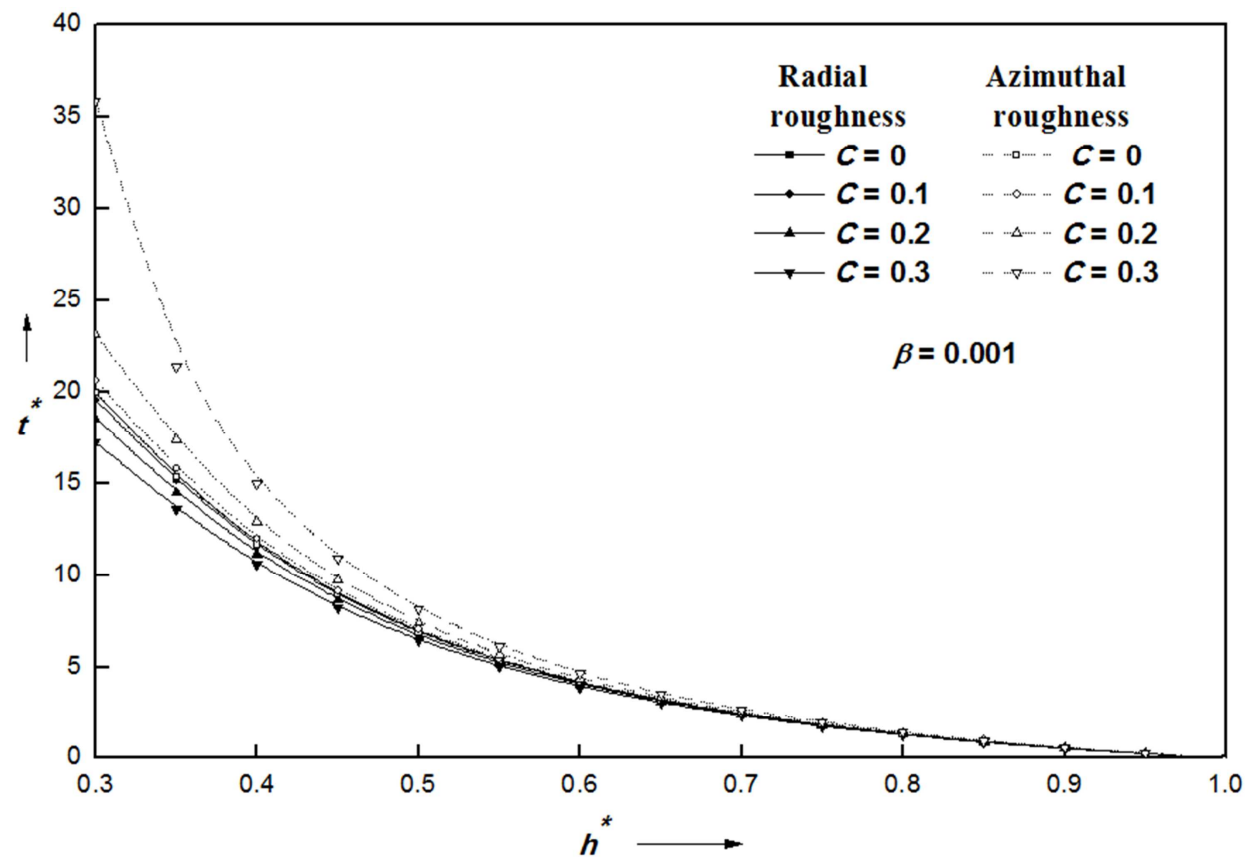

Figure 7. Variation of squeeze film time $t^{*}$ with film thickness $h^{*}$ for different values of $C$ with $\beta=0.001$.

\section{Conclusions}

In the present analysis, the combined effect of surface roughness and non-Newtonian lubricants on squeeze film characteristics between rough circular plates is presented on the basis of Rabinowitsch fluid model and Christensen's roughness model. The modified Reynolds equations for radial and azimuthal roughness are derived. An analytic solution for film pressure and squeezing time are obtained by means of small perturbation technique.

It is analysed that in comparison to the Newtonian lubricants, dimensionless film pressure, load carrying capacity and squeeze film time are smaller for pseudoplastic lubricants, while higher values of these properties are obtained for dilatant lubricants.

Film pressure, load carrying capacity and squeeze film time decrease with the increase in pseudoplasticity, while the results are reversed for an increase in dilatancy. In comparison to the smooth surfaces $(c=0)$, the film pressure, load capacity and squeezing time is smaller for radial roughness and greater for azimuthal roughness. In the case of radial roughness, the film pressure, load capacity and squeezing time decreases with an increase in surface roughness, while the results are reversed in case of azimuthal roughness.

Based on the present analysis, it is concluded that the stability and load sustaining capacity of a squeezing film bearing can be increased by reducing the radial roughness, introducing optimal azimuthal roughness and blending the proper additives to the lubricant to make it dilatant up to an optimal level.

\section{Nomenclature}

C dimensionless roughness parameter

E expectancy operator
$\mathrm{H} \quad$ non-dimensional film thickness

$\mathrm{H}_{\mathrm{i}} \quad$ film thickness

$\mathrm{h}_{\mathrm{s}} \quad$ stochastic film thickness

$\mathrm{k}$ non-linear factor of lubricants

L bearing length

$\mathrm{P} \quad$ pressure in the film region

$\mathrm{P}^{*} \quad$ non-dimensional pressure

$\mathrm{R}$ radius of the circular plate

$\mathrm{r}, \theta \quad$ radial and axial coordinates, respectively

$\mathrm{t}$ time of approach

$t^{*} \quad$ non-dimensional time of approach

V squeezing velocity

$\mathrm{u}, \mathrm{w}$ velocity components of lubricant in the $\mathrm{x}$ - and $\mathrm{y}$ -

directions, respectively

W load carrying capacity

$\mathrm{W}^{*} \quad$ non-dimensional load carrying capacity

$\mathrm{x}, \mathrm{y}$ horizontal and vertical coordinates

$\beta \quad$ dimensionless non-linear factor of lubricants

$\mu \quad$ initial viscosity

$\tau_{\mathrm{xy}} \quad$ shear stress component

\section{References}

[1] Singh UP, Gupta RS, Kapur VK. On the application of Rabinowitsch fluid model on an annular ring hydrostatic thrust bearing. Tribol Int 2013; 58: 65-70.

[2] Tian Z, Cao H, Huang Y. Static characteristics of hydrostatic thrust bearing considering the inertia effect on the region of supply hole. Proc Inst Mech Eng Part J J Eng Tribol 2018.

[3] Bakker OJ, van Ostayen R a. J. Recess Depth Optimization for Rotating, Annular, and Circular Recess Hydrostatic Thrust Bearings. J Tribol 2010. doi: 10.1115/1.4000545. 
[4] Jurczak P, Falicki J. Pressure distribution in a squeeze film spherical bearing with rough surfaces lubricated by an ellis fluid. Int J Appl Mech Eng 2016. doi: 10.1515/ijame-2016-0036.

[5] Walicka A, Walicki E, Jurczak P, Falicki J. Curvilinear squeeze film bearing with porous wall lubricated by a Rabinowitsch fluid. Int J Appl Mech Eng 2017. doi: 10.1515/-0026.

[6] Singh UP. Combined effects of piezo-viscosity and couple stress fluids on squeeze film between circular plates. Int J Fluid Mech Res 2014; 41. doi: 10.1615/InterJFluidMechRes.v41.i4.60.

[7] Naduvinamani NB, Apparao S, Kadadi AK, Biradar SN Combined Effects of Viscosity Variation and Surface Roughness on the Squeeze Film Lubrication of Journal Bearings with Micropolar Fluids. Tribol Online 2014. doi: 10.2474/trol.9.175.

[8] Singh UP, Gupta RS, Kapur VK. On the steady performance of hydrostatic thrust bearing: Rabinowitsch fluid model. Tribol Trans 2011; 54: 723-9. doi: 10.1080/10402004.2011.597541.

[9] Singh, U. P., Gupta, R. S., Kapur VK. On the Steady Performance of Annular Hydrostatic Thrust Bearing: Rabinowitsch Fluid Model. J Tribol 2012; 134: 1-5.

[10] Singh UP, Gupta RS, Kapur VK. On the squeeze film characteristics between a long cylinder and a flat plate: Rabinowitsch model. Proc Inst Mech Eng Part J J Eng Tribol 2013; 227. doi: $10.1177 / 1350650112458742$.

[11] Singh UP, Gupta RS, Kapur VK. Non-Newtonian Effects on the Squeeze Film Characteristics between a Sphere and A Flat Plate: Rabinowitsch Model. Adv Tribol 2012; 2012: 1-7. doi: DOI: 10.1155 / 2012 / 571036.

[12] Wada S, Hayashi H. Hydrodynamic Lubrication of Journal Bearings by Pseudo-Plastic Lubricants: Part 2, Experimental Studies. Bull JSME 1971. doi: 10.1299/jsme1958.14.279.

[13] Hsu YC, Saibel E. Slider bearing performance with a non-newtonian lubricant. ASLE Trans 1965; 8: 191-4. doi: $10.1080 / 05698196508972093$.

[14] Sharma SC, Jain SC, Sah PL. Effect of non-Newtonian behaviour of lubricant and bearing flexibility on the performance of slot-entry journal bearing. Tribol Int 2000; 33: 507-17. doi: 10.1016/S0301-679X (00) 00093-1.

[15] Sinhasan R, Sah PL. Static and dynamic performance characteristics of an orifice compensated hydrostatic journal bearing with non-Newtonian lubricants. Tribol Int 1996; 29: 515-26. doi: https://doi.org/10.1016/0301-679X (95) 00115-K.
[16] Bourging P, Gay B. Determination of the load capacity of finite width journal bearing by finite element method in the case of a non-Newtonian lubricant. J Tribol 1984; 106: 285-90.

[17] Hashimoto H. Non-Newtonian effects on the static characteristics of one dimensional slider bearings in the inertial flow regime. J Tribol 1994; 116: 303-9.

[18] Lin J-R. Non-Newtonian effects on the dynamic characteristics of one dimensional slider bearings: Rabinowitsch model. Tribol Lett 2001; 10: 237-43.

[19] Singh UP. Application of rabinowitsch fluid model to pivoted curved slider bearings. Arch Mech Eng 2013. doi: 10.2478/meceng-2013-0016.

[20] Singh UP, Gupta RS, Kapur VK. Effects of inertia in the steady state pressurised flow of a non-Newtonian fluid between two curvilinear surfaces of revolution: Rabinowitsch fluid model. Chem Process Eng - Inz Chem i Proces 2011; 32. doi: 10.2478/v10176-011-0027-1.

[21] Singh UP, Gupta RS, Kapur VK. On the performance of pivoted curved slider bearings: Rabinowitsch fluid model. Tribol Ind 2012; 34.

[22] Singh UP, Medhavi A, Gupta RS, Bhatt SS. Theoretical study of heat transfer on peristaltic transport of Non-Newtonian fluid flowing in a channel: Rabinowitsch fluid model. Int J Math Eng Manag Sci 2018; 3.

[23] Singh UP, Medhavi A, Gupta RS, Bhatt SS. Analysis of Peristaltic Transport of Non-Newtonian Fluids Through Nonuniform Tubes: Rabinowitsch Fluid Model. Zeitschrift Fur Naturforsch - Sect A J Phys Sci 2017; 72. doi: 10.1515/zna-2017-0033.

[24] Bhatt SS, Medhavi A, Gupta RS, Singh UP. Effects of Heat Transfer during Peristaltic Transport in Nonuniform Channel with Permeable Walls. J Heat Transfer 2017; 139. doi: $10.1115 / 1.4034551$.

[25] Walicka A, Walicki E, Jurczak P, Falicki J. Curvilinear squeeze film bearing with rough surfaces lubricated by a RabinowitschRotem-Shinnar fluid. Appl Math Model 2016; 40: 7916-27. doi: 10.1016/j.apm.2016.03.048.

[26] Christensen H. Stochastic Models for Hydrodynamic Lubrication of Rough Surfaces. Proc Inst Mech Eng 1969. doi: 10.1243/PIME_PROC_1969_184_074_02. 